\title{
ON RESIDUALLY FINITE GROUPS AND THEIR GENERALIZATIONS
}

BY

\section{ANDRZEJ STROJNOWSKI（WARSZAWA）}

The paper is concerned with the class of groups satisfying the finite embedding (FE) property. This is a generalization of residually finite groups. In [2] it was asked whether there exist FE-groups which are not residually finite. Here we present such examples. To do this, we construct a family of three-generator soluble FE-groups with torsion-free abelian factors. We study necessary and sufficient conditions for groups from this class to be residually finite. This answers the questions asked in [1] and [2].

1. The construction of the group $G(\phi)$. Let $\phi$ be a map from $\mathbb{Z}$ into $\mathbb{Z} \backslash\{0\}$. We define $G(\phi)$ to be the group generated by elements $\left\{x_{i}\right\}_{i \in \mathbb{Z}} \cup\left\{y_{j}\right\}_{j \in \mathbb{Z}} \cup\{z\}$ with the following relations:

$$
\left[x_{i}, x_{j}\right]=\left[y_{i}, y_{j}\right]=1, z^{-1} x_{i} z=x_{i-1}, z^{-1} y_{j} z=y_{j-1}, y_{j}^{-1} x_{i} y_{j}=x_{i}^{\phi(i-j)} .
$$

It is obvious that the group $G(\phi)$ is generated by three elements $x=x_{0}$, $y=y_{0}$ and $z$.

Let us start with a lemma describing the abelian subgroups of $G(\phi)$.

LEMMA 1.1. Let $H$ be a normal subgroup of a group $G$ and let $h \in H$ be an element of infinite order. Assume we are given a set $S$ consisting of integers $s$ such that $h$ is conjugate to $h^{s} \in G$. For each $s \in S$ we choose an element $y_{s} \in G$ such that $y_{s}^{-1} h y_{s}=h^{s}$. Let $Y$ denote the subgroup of $G$ generated by the set $\left\{y_{s}\right\}_{s \in S}$ and let $C$ be the multiplicative semigroup generated by $S$. Then:

(i) There exists a subgroup $A$ of $H$ such that $h \in A$ and $A$ is isomorphic to the additive group of $\mathbb{Z} C^{-1}$.

(ii) For any $y$ in $Y$ there exist $a$ and $b$ in $C$ such that $y^{-1} h^{a} y=h^{b}$.

(iii) For any $a$ and $b$ in $C$ there exists $y$ in $Y$ such that $y^{-1} h^{a} y=h^{b}$.

(iv) If $Y$ is abelian then the subgroup $A$ of $H$ generated by $\left\{y^{-1} h y\right.$ : $y \in Y\}$ is isomorphic to the additive group of $\mathbb{Z} C^{-1}$.

1991 Mathematics Subject Classification: Primary 20E26.

Supported by KBN grant No 211159101. 
Proof. (i) Let $c_{1}, c_{2}, c_{3}, \ldots$ be the list of all elements of $C$. By induction we can construct a sequence $h_{0}, h_{1}, h_{2}, \ldots$ of elements of $H$ such that $h_{0}=h, h_{n}^{c_{n}}=h_{n-1}$ and each $h_{n}$ is conjugate in $G$ to $h$. The subgroup $A=\left\langle h_{0}, h_{1}, h_{2}, \ldots\right\rangle$ of $H$ is clearly isomorphic to the additive group of $\mathbb{Z} C^{-1}$

(ii) We proceed by induction on the length of the word $y$ written in the letters $y_{s}$.

If $y=y_{s}$ we set $a=s$ and $b=s^{2}$, if $y=y_{s}^{-1}$ we set $a=s^{2}$ and $b=s$.

Let $y=y_{s} z$ or $y=y_{s}^{-1} z$ where $z$ is an element of $Y$ of smaller length. By induction, there exist $a$ and $b$ in $C$ such that $z^{-1} h^{a} z=h^{b}$. Now we have $y^{-1} h^{a} y=h^{b s}$ or $y^{-1} h^{a s} y=h^{b}$.

(iii) There exist $g$ and $x$ in $Y$ such that $g^{-1} h g=h^{a}$ and $x^{-1} h x=h^{b}$. Therefore, $\left(g^{-1} x\right)^{-1} h^{a} g^{-1} x=h^{b}$.

(iv) By (i), it is sufficient to prove that for all $y \in Y$ the element $y^{-1} h y$ belongs to the subgroup $A=\left\langle h_{0}, h_{1}, h_{2}, \ldots\right\rangle$. Take some $y \in Y$. By (ii), there exist $c_{n}$ and $c_{t}$ in $C$ such that $y^{-1} h^{c_{n}} y=h^{c_{t}}$. By (i), there exists $z \in Y$ such that $z^{-1} h z=h_{n}$. Since $Y$ is abelian and $h=h_{n}^{c_{n} c_{n-1} \ldots c_{1}}$, we get

$$
y^{-1} h_{n}^{c_{n}} y=\left(y^{-1} z^{-1} h z y\right)^{c_{n}}=z^{-1} y^{-1} h^{c_{n}} y z=z^{-1} h^{c_{t}} z=h_{n}^{c_{t}} .
$$

Hence,

$$
y^{-1} h y=\left(y^{-1} h_{n}^{c_{n}} y\right)^{c_{n-1} \ldots c_{2} c_{1}}=h_{n}^{c_{t} c_{n-1} \ldots c_{1}} \in A .
$$

Notation. Similarly to Lemma 1.1 , for the group $G(\phi)$ we will denote by $C$ the subsemigroup of $\mathbb{Z}$ generated by $\operatorname{im} \phi$.

Proposition 1.2. Every element of the group $G(\phi)$ can be uniquely written as a finite product

$$
\prod_{i \in \mathbb{Z}} x_{i}^{\alpha(i)} \cdot \prod_{j \in \mathbb{Z}} y_{j}^{\beta(j)} \cdot z^{t}
$$

where $t \in \mathbb{Z}$ and $\alpha(i) \in \mathbb{Z} C^{-1}, \beta(j) \in \mathbb{Z}$ for all integers $i, j$.

Proof. By Lemma 1.1(iv), the subgroup $X_{i}=\left\langle y_{j} x_{i} y_{j}^{-1}: j \in \mathbb{Z}\right\rangle$ is isomorphic to the additive group of $\mathbb{Z} C^{-1}$. Now it is sufficient to use the fact that $z^{-1} X_{i} z=X_{i-1}$.

Let $X$ be the normal subgroup of $G(\phi)$ generated by $x=x_{0}$ and let $Y$ be the normal subgroup of $G(\phi)$ generated by $x=x_{0}$ and $y=y_{0}$. These definitions yield:

COROLlarY 1.3. There exist normal subgroups $X$ and $Y$ of $G(\phi)$ such that $X$ is isomorphic to the infinite product of the additive group $\mathbb{Z} C^{-1}$, and $Y / X, G(\phi) / Y$ are free abelian groups. 
2. Residually finite groups. In this section we describe some conditions for the group $G(\phi)$ to be residually finite.

Definition. We will say that a group $G$ is approximated by finite $p$ groups if for every $1 \neq g \in G$ there exists a normal subgroup $H$ of $G$ such that $g \notin H$ and the index of $H$ in $G$ is $p^{n}$ for some $n$.

Clearly, if $G$ is approximated by finite $p$-groups then $G$ is approximated by finite groups and so $G$ is a residually finite group.

Consider the following two simple examples.

ExAmPLE 2.1. Let $\phi(i)=1$ for all i. Then $G(\phi)$ is approximated by finite $p$-groups for any prime $p$. This is clear since $G(\phi)$ is a wreath product of the free abelian group generated by $x$ and $y$ by the infinite cyclic group generated by $z$.

EXAMPLE 2.2. Let $\phi$ be a map onto the set of all primes. Then $G(\phi)$ contains subgroups isomorphic to the additive group of rational numbers so it is not residually finite.

This example was described by P. Hall in [5], Theorem 2. He proved that this is a minimal example (in the sense of minimal soluble rank) of a soluble group which is not residually finite. Moreover, this group contains a maximal subgroup of infinite index. See also [9], Theorem 9.58.

LEMMA 2.3. Let $H$ be the normal subgroup of $G(\phi)$ generated by $z^{n}$ and $y^{m}$. Then $H$ consists of finite products

$$
\prod_{i \in \mathbb{Z}} x_{i}^{\alpha(i)} \cdot \prod_{j \in \mathbb{Z}} y_{j}^{\beta(j)} \cdot z^{n t},
$$

where $\alpha(i) \in \mathbb{Z} C^{-1}$ and $\sum_{i \in \mathbb{Z}} \alpha(i n+k)$ belongs to the ideal $J(n, m)$ of $\mathbb{Z} C^{-1}$ generated by the integers $\phi(j)-\phi(j-n)$ and $\phi(j)^{m}-1$ for all $j$. Moreover, $\beta(j) \in \mathbb{Z}$ and $\sum_{j \in \mathbb{Z}} \beta(j n+k) \in m \mathbb{Z}$ for all integers $k$.

Proof. We have $z^{n} \in H$ so $H$ contains also

$$
x_{i} x_{i+n}^{-1}=x_{i} z^{n} x_{i}^{-1} z^{-n} \quad \text { and } \quad y_{j} y_{j+n}^{-1}=y_{j} z^{n} y_{j}^{-1} z^{-n},
$$

for all integers $i$ and $j$. Consequently, $H$ contains

$$
x_{i}^{\phi(j)-\phi(j-n)}=x_{i}^{\phi(j)} y_{i-j} y_{i-j+n}^{-1} x_{i}^{-\phi(j)} y_{i-j+n} y_{i-j}^{-1}
$$

and

$$
x_{i}^{\phi(j)^{m}-1}=x_{i}^{-1} y_{i-j}^{-m} x_{i} y_{i-j}^{m} .
$$

Let $k \in J(n, m)$. Then there exists an integer $c \in C$ such that $c k$ is a sum of integers of the form $\phi(j)-\phi(j-n)$ or $\phi(j)^{m}-1$. Then $x_{i}^{c k}$ is a product of $x_{i}^{\phi(j)-\phi(j-n)}, x_{i}^{\phi(j)^{m}-1}$ and their inverses. By Lemma 1.1, there exists $y \in Y$ such that $y^{-1} x_{i} y=x_{i}^{c}$. This yields $x_{i}^{k} \in H$. Using elements of the form $x_{i} x_{i+n}^{-1}$, we can prove that a finite product $\prod_{i \in \mathbb{Z}} x_{i}^{\alpha(i)}$ belongs to 
$H$, where $\alpha(i) \in \mathbb{Z} C^{-1}$ and $\sum_{i \in \mathbb{Z}} \alpha(i n+k)$ belongs to the ideal $J(n, m)$ of $\mathbb{Z} C^{-1}$. Similarly we can prove that for all integers $k$, the product $\prod_{j \in \mathbb{Z}} y_{j}^{\beta(j)}$ belongs to $H$, where $\prod_{j \in \mathbb{Z}} \beta(j n+k) \in m \mathbb{Z}$. To end the proof, one can easily check that the subgroup defined above is stable under conjugations by $x, y$ and $z$.

THEOREM 2.4. Let $\phi$ be a map from $\mathbb{Z}$ into $\mathbb{Z} \backslash\{0\}$. Let $C$ be the multiplicative semigroup generated by the image of $\phi$. Then the group $G(\phi)$ is residually finite if and only if for any positive integer $N$ there exist integers $m>N, n>N$ and $t>N$ such that $t \notin C$ and the ideal $J(n, m)$ of $\mathbb{Z} C^{-1}$ generated by the set $\left\{\phi(j)-\phi(j-n), \phi(j)^{m}-1: j \in \mathbb{Z}\right\}$ is contained in $t \mathbb{Z} C^{-1}$.

Proof. $\Rightarrow$ Suppose $G(\phi)$ is residually finite. Take an integer $N>0$. Then there exists a normal subgroup $H$ of $G(\phi)$ such that $z^{i}, y^{i}$ and $x^{i}$ do not belong to $H$ for $i \leq N$. Let $n, m$ and $t$ be the smallest positive integers such that $H$ contains $z^{n}, y^{m}$ and $x^{t}$. By Lemma 2.3, $H$ contains $x^{j}$ for all $j \in J(n, m)$. Hence $J(n, m) \subset t \mathbb{Z} C^{-1}$.

$\Leftarrow$ Fix a positive integer $N$. Let $m, n, t>N$ be integers such that $t \notin C$ and $J(n, m) \subset t \mathbb{Z} C^{-1}$. Let $H_{N}$ be the normal subgroup generated by $z^{n}, y^{m}$ and $x^{t}$. Then by Lemma 2.3 , the subgroup $H_{N}$ consists of finite products

$$
\prod_{i \in \mathbb{Z}} x_{i}^{\alpha(i)} \cdot \prod_{j \in \mathbb{Z}} y_{j}^{\beta(j)} \cdot z^{n s},
$$

where $\alpha(i) \in \mathbb{Z} C^{-1}, \sum_{i \in \mathbb{Z}} \alpha(i n+k) \in t \mathbb{Z} C^{-1}, \beta(j) \in \mathbb{Z}$ and $\sum_{j \in \mathbb{Z}} \beta(j n+k) \in$ $m \mathbb{Z}$ for all integers $k$. This subgroup has a finite index equal to $n m^{n} t^{n}$. It is clear that the intersection of the subgroups $H_{N}$ over all positive integers $N$ is trivial. Hence $G(\phi)$ is residually finite.

THEOREM 2.5. Let $\phi: \mathbb{Z} \rightarrow \mathbb{Z} \backslash\{0\}$ be periodic with period $n$ (that is, $\phi(n+i)=\phi(i)$ for all $i \in \mathbb{Z})$. Then $G(\phi)$ is residually finite.

Proof. Suppose $p$ is a prime with does not divide any of $\phi(1), \ldots, \phi(n)$ where $n$ is the period of $\phi$. Let $G p$ be the normal subgroup of $G(\phi)$ generated by $z^{n p}, y^{p-1}$ and $x^{p}$. Since $p$ divides $\phi(i)^{p-1}-1$ for all $i$, by Lemma 2.3 the group $G p$ consists of elements of the form

$$
\prod_{i \in \mathbb{Z}} x_{i}^{\alpha(i)} \cdot \prod_{j \in \mathbb{Z}} y_{j}^{\beta(j)} \cdot z^{p n},
$$

where $\sum_{k \in \mathbb{Z}} \alpha(i+p k) \in p \mathbb{Z} C^{-1}$ for all $i$ and $\sum_{k \in \mathbb{Z}} \beta(j+p k) \in(p-1) \mathbb{Z}$ for all $j$. One can easily check that the index of $G p$ in $G(\phi)$ is $n p(p-1)^{n p} p^{n p}$. It is clear that the intersection of all subgroups $G p$, for $p$ prime not dividing any of $\phi(1), \ldots, \phi(n)$, is trivial. 
THEOREM 2.6. Let $p$ be a prime. Then $G(\phi)$ is approximated by finite $p$ groups if and only if $p \notin C$ and for any positive integer $N$ there exist integers $m>N, n>N$ and $t>N$ such that the ideal $J\left(p^{n}, p^{m}\right)$ is contained in $p^{t} \mathbb{Z} C^{-1}$.

Proof. $\Rightarrow$ Suppose $G(\phi)$ is approximated by finite $p$-groups. Let $N>0$ be an integer. Then there exists a normal subgroup $H$ of $G(\phi)$ such that $G(\phi) / H$ is a finite $p$-group and $z^{i}, y^{i}$ and $x^{i}$ do not belong to $H$ for $i \leq p^{N}$. Let $n, m$ and $t$ be the smallest positive integers such that $H$ contains $z^{n}, y^{m}$ and $x^{t}$. By Lemma 2.3, $H$ contains $x^{j}$ for all $j \in J(n, m)$. Hence $J(n, m) \subset$ $t \mathbb{Z} C^{-1}$. Furthermore, $n, m$ and $t$ are some powers of $p$ since the index of $H$ is a power of $p$.

$\Leftarrow$ Let $H_{N}$ be a normal subgroup of $G(\phi)$ defined in the following way: Let $m, n, t>N$ be integers such that $J\left(p^{n}, p^{m}\right) \subset p^{t} \mathbb{Z} C^{-1}$. Let $H_{N}$ be the normal subgroup generated by $z^{p^{n}}, y^{p^{m}}$ and $x^{p^{t}}$. Then by Lemma $2.3, H_{N}$ consists of finite products

$$
\prod_{i \in \mathbb{Z}} x_{i}^{\alpha(i)} \cdot \prod_{j \in \mathbb{Z}} y_{j}^{\beta(j)} \cdot z^{s p^{n}},
$$

where $\alpha(i) \in \mathbb{Z} C^{-1}, \sum_{i \in \mathbb{Z}} \alpha(i n+k) \in p \mathbb{Z} C^{-1}, \beta(j) \in \mathbb{Z}$ and $\sum_{j \in \mathbb{Z}} \beta(j n+$ $k) \in p^{m} \mathbb{Z}$ for all integers $k$. This subgroup has a finite index equal to $p^{n+m p^{n}+t p^{n}}$. It is clear that the intersection of all subgroups $H_{N}$ over all positive integers $N$ is trivial. Hence $G(\phi)$ is residually finite.

TheOREM 2.7. Let $m>1$. Let $\phi: \mathbb{Z} \rightarrow \mathbb{Z}$ be defined by $\phi(i)=i m+1$. Then $G(\phi)$ is approximated by finite p-groups if and only if the prime $p$ divides $m$.

Pr o of. $\Rightarrow$ Suppose that $p$ does not divide $m$. Then there exists an integer $i$ such that $p$ divides $i m+1$. Hence $p \in C$ and consequently $G(\phi)$ is not approximated by finite $p$-groups.

$\Leftarrow$ Suppose $p$ divides $m$. Let $n$ be a positive integer. Then the ideal $J\left(p^{n}, p^{n}\right)$ is generated by

$$
\phi(j)-\phi\left(j-p^{n}\right)=j m+1-\left(j-p^{n}\right) m-1=p^{n} m
$$

and by

$$
\phi(j)^{p^{n}}-1=(j m+1)^{p^{n}}-1 .
$$

One can easily show by induction on $n$ that all these elements belong to $p^{n} \mathbb{Z}$. This yields $J\left(p^{n}, p^{n}\right) \subset p^{n} \mathbb{Z} C^{-1}$. By Theorem 2.6, $G(\phi)$ is approximated by finite $p$-groups.

Now we show that the residual finiteness of $G(\phi)$ does not depend on the semigroup $C$. 
EXAMPLE 2.8. Let $m>0$ be an integer and let $\phi(i)=m$ for all $i$. Then $G(\phi)$ is approximated by finite $p$-groups for all primes $p$ relatively prime to $m$.

EXAMPLE 2.9. Let $\phi(i)=1$ for $i \neq 0$ and $\phi(0)=m$, where $m>1$ is an integer. Then $G(\phi)$ is not residually finite.

P r o of. Suppose $H$ is a normal subgroup of $G(\phi)$ of a finite index. Then $H$ contains $z^{n}$ for some $n$. This yields

$$
y_{0} y_{n}^{-1}=y_{0} z^{n} y_{0}^{-1} z^{-n} \in H .
$$

Consequently, $H$ contains

$$
x_{0}^{m-1}=x_{0}^{\phi(0)-\phi(n)}=x_{0}^{\phi(0)} y_{0} y_{n}^{-1} x_{0}^{-\phi(0)} y_{n}^{-1} y_{0} .
$$

Hence $G(\phi)$ is not residually finite.

\section{Groups with the finite embedding property}

Definition. Following [3], we will say that a group $G$ is a Finite Embedding group (FE-group) if for every finite subset $X$ of $G$ there exists an injection $\Psi$ of $X$ into a finite group $H$ such that if $x, y$ and $x y$ are in $X$ then

$$
\Psi(x y)=\Psi(x) \Psi(y) .
$$

TheOREM 3.1 ([3], Proposition 1.2). All residually finite groups are FEgroups.

THEOREM 3.2. Every finitely related FE-group $G$ is residually finite.

Proof. Let $G$ be a FE-group generated by a set $S$ with relations $r_{1}, \ldots$ $\ldots, r_{n}$. Then $G=F(S) / R$ where $F(S)$ is the free group generated by $S$ and $R$ is the normal subgroup of $F(S)$ generated by the set of relations. Let $\phi: F(S) \rightarrow G$ be the canonical projection. Let $v \neq 1$ be an element of $G$ and $w \in F(S)$ be such that $\phi(w)=v$. Let $X$ be the set of all subwords of $w, r_{1}, \ldots, r_{n}$ including the empty word. By definition, there exists an injection $\Psi$ of $\phi(X)$ into a finite group $H$ such that if $x, y$ and $x y$ are in $\phi(X)$ then

$$
\Psi(x y)=\Psi(x) \Psi(y) .
$$

Let $\Lambda: F(S) \rightarrow H$ be the group homomorphism given by

$$
\Lambda(s)= \begin{cases}\Psi(\phi(s)) & \text { if } s \in X \cap S, \\ 1 & \text { if } s \in S \backslash X .\end{cases}
$$

We arrive at a commutative diagram of group morphisms:

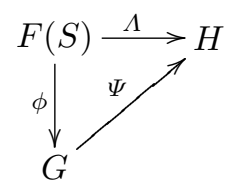


By the properties of $\Psi$, the set $\left\{r_{1}, \ldots, r_{n}\right\}$ of relations is contained in ker $\Lambda$. Hence we can extend $\Psi$ to a group homomorphism $\lambda: G \rightarrow H$. Since $\Psi$ is an injection, $\lambda(v) \neq 1$. Furthermore, $\operatorname{ker} \lambda$ is a subgroup of $G$ of finite index.

Proposition 3.3. Let $G$ be a group such that for every finite subset $X$ of $G$ there exists an injection $\Psi$ of $X$ into a residually finite group $\Gamma$ such that if $x, y$ and $x y$ are in $X$ then $\Psi(x y)=\Psi(x) \Psi(y)$. Then $G$ is a FE-group.

Proof. Let $X, \Psi$ and $\Gamma$ be as in the assumptions. Since $\Psi(X)$ is a finite subset of $\Gamma$, there exists an injection $\tau$ of $\Psi(X)$ into a finite group $H$ such that if $x, y$ and $x y$ are in $X$ then $\tau(\Psi(x y))=\tau(\Psi(x)) \tau(\Psi(y))$. Now $\tau \circ \Psi: X \rightarrow H$ is the required injection.

The aim of this section is to prove that $G(\phi)$ is a FE-group for every $\phi$. This gives us a series of not residually finite FE-groups.

THEOREM 3.4. The group $G(\phi)$ satisfies the FE condition for all functions $\phi$.

Proof. Let $\phi: \mathbb{Z} \rightarrow \mathbb{Z} \backslash\{0\}$ and let $X$ be a finite subset of $G(\phi)$. Then there exists a positive integer $n$ such that all elements of $X$ can be written as products

$$
\prod_{i=-n}^{n} x_{i}^{\alpha(i)} \cdot \prod_{j=-n}^{n} y_{j}^{\beta(j)} \cdot z^{t}
$$

where for all $i$ and $j$ we have $\alpha(i) \in \mathbb{Z} C^{-1}, \beta(j) \in \mathbb{Z}$ and $-n \leq t \leq n$. The multiplication in $X$ looks as follows:

$$
\begin{aligned}
& \prod_{i=-n}^{n} x_{i}^{\alpha(i)} \cdot \prod_{j=-n}^{n} y_{j}^{\beta(j)} \cdot z^{t} \cdot \prod_{i=-n}^{n} x_{i}^{\delta(i)} \cdot \prod_{j=-n}^{n} y_{j}^{\gamma(j)} \cdot z^{k} \\
& =\prod_{i=-n}^{n} x_{i}^{\alpha(i)} \cdot \prod_{j=-n}^{n} y_{j}^{\beta(j)} \cdot \prod_{i=-n}^{n} x_{i+t}^{\delta(i)} \cdot \prod_{j=-n}^{n} y_{j+t}^{\gamma(j)} \cdot z^{t+k} \\
& =\prod_{i=-n}^{n+t} x_{i}^{\alpha(i)+\delta(i-t)} \prod_{j=-n}^{n} \phi(j+t-i)^{\beta(j)} \cdot \prod_{j=-n}^{n+t} y_{j}^{\beta(j)+\gamma(j-t)} \cdot z^{t+k} .
\end{aligned}
$$

Let $\psi: \mathbb{Z} \rightarrow \mathbb{Z} \backslash\{0\}$ be a periodic function with period $6 n+2$ defined by

$$
\psi(i)= \begin{cases}\phi(i) & \text { for }-3 n \leq i \leq 3 n \\ M & \text { for } i=3 n+1\end{cases}
$$

where $M$ is an integer so large that every element of $X$ can be considered as an element of $G(\phi)$. Let $\lambda: X \rightarrow G(\psi)$ be the injection given by

$$
\lambda\left(\prod_{i=-n}^{n} x_{i}^{\alpha(i)} \cdot \prod_{j=-n}^{n} y_{j}^{\beta(j)} \cdot z^{t}\right)=\prod_{i=-n}^{n} x_{i}^{\alpha(i)} \cdot \prod_{j=-n}^{n} y_{j}^{\beta(j)} \cdot z^{t}
$$


It is clear that $\lambda(a b)=\lambda(a) \lambda(b)$ for $a, b \in X$. Since by Theorem 2.5, $G(\psi)$ is residually finite, it is a FE-group by Proposition 3.3.

COROLLARY 3.5. There exists a finitely generated FE-group which is not locally residually finite.

Proof. Let $\phi$ be a function from $\mathbb{Z}$ onto the set of all primes. Then $G(\phi)$ is generated by 3 elements, it is not residually finite since it contains subgroups isomorphic to the additive group of $\mathbb{Q}$ and by Theorem 3.3, it is a FE-group.

4. Idempotents. One of the famous open problems in group theory is the following one formulated by Kaplansky [6]:

CONJECTURE. The group algebra $k[G]$ of a torsion free group $G$ over a field has no nontrivial idempotents.

Formanek [4] gave a partial answer to this conjecture in the case when $K$ is a field of characteristic 0 and for groups satisfying the following nondivisibility condition:

(*) $\quad$ For each $1 \neq g \in G$ there are infinitely many primes $p$ such that $g$ is not conjugate to any of $g^{p}, g^{p^{2}}, g^{p^{3}}, \ldots$

Zalesskiu and Mikhalev [8] studied idempotents in group algebras of positive characteristic $p$ and formulated the following condition:

$\left(\mathrm{D}_{p}\right) \quad$ For any $g \in G$, if $g$ is conjugate to $g^{p^{N}}$ for some integer $N>0$ then $g$ has finite order.

In [1] Bass reformulated the condition (*) follows:

(D) Suppose $H$ is a finitely generated subgroup of $G, g \in G, N$ is an integer $>0$ and for all but finitely many primes $p, g$ is conjugate in $H$ to $g^{p^{N}}$. Then $g$ has finite order.

He proved that linear groups satisfy condition (D) and the torsion free linear groups satisfy Kaplansky's Conjecture. He also proved that the (D)groups satisfy the following conjecture:

BAss' Strong Conjecture [1]. Let $P$ be a finitely generated projective module over the integral group ring $\mathbb{Z}[G]$. Then $r_{p}(g)=0$ for $g \neq 1$, where $r_{p}$ is the trace map.

Strojnowski [10] proved Bass' Strong Conjecture for groups satisfying the following condition:

(WD) Suppose $H$ is a finitely generated subgroup of $G, g \in H, N$ is an integer $>0$ and for all primes $p, g$ is conjugate to $g^{p^{N}}$. Then $g=1$. 
In this paper we give a series of examples to show how these conditions differ.

THEOREM 4.1. (i) $G(\phi)$ satisfies condition $\left(\mathrm{D}_{p}\right)$ if and only if the group $C C^{-1}$ does not contain any power of the prime $p$.

(ii) $G(\phi)$ satisfies condition (D) if and only if for all integers $N>0$ the group $C C^{-1}$ does not contain infinitely many elements of the set $\left\{p^{N}: p\right.$ is a prime $\}$.

(iii) $G(\phi)$ satisfies condition (WD) if and only if for any integer $N>0$ there exists a prime number $p$ such that $p^{N}$ does not belong to the group $C C^{-1}$.

P r o of. Since the proofs of all parts are similar we only show (i). Let $p^{n} \in$ $C C^{-1}$. Then by Lemma 1.1(iii), there exists an element $g$ of the subgroup generated by all $y_{s}$ such that $g^{-1} x g=x^{p^{N}}$. Hence $G(\phi)$ does not satisfy condition $\left(\mathrm{D}_{p}\right)$.

Conversely, if $G(\phi)$ does not satisfy $\left(\mathrm{D}_{p}\right)$ then there exists $h \in G(\phi)$ of infinite order such that $h$ is conjugate to its $p^{n}$ th power. Since the groups $G(\phi) / Y$ and $Y / X$ are free abelian, they do not contain the additive group $\mathbb{Z}[1 / p]$. Hence by Lemma $1.1(\mathrm{i}), h \in X$. Let $h=\prod_{i=a}^{b} x_{i}^{\alpha(i)}$ and let $g=$ $\prod_{j=c}^{d} y_{j}^{\beta(j)} \cdot z^{t} \in G(\phi)$ be such that $g^{-1} h g=h^{p N}$. Then

$$
h^{p N}=\prod_{i=a}^{b} x_{i}^{\alpha(i) p^{n}}=z^{-t}\left(\prod_{i=a}^{b} x_{i}^{\alpha(i)} \prod_{j=c}^{d} \phi(i-j)^{\beta(j)}\right) z^{t} .
$$

Hence $t=0$ and for all $i$, if $\alpha(i) \neq 0$ then $\prod_{j=c}^{d} \phi(i-j)^{\beta(j)}=p^{n}$. Thus, $p^{n} \in C C^{-1}$.

EXAMPLE 4.2. Let $\phi$ be a map from the integers onto the set $\left\{p^{p}: p\right.$ is a prime . Then $G(\phi)$ satisfies conditions (D) and (WD) but does not satisfy $(*)$ or $\left(\mathrm{D}_{p}\right)$ for any prime $p$.

EXAMPLE 4.3. Let $\phi$ be a map from the integers onto $\{2 p: p$ is an odd prime\}. Then $G(\phi)$ satisfies (WD), (D), $(*)$, and $\left(\mathrm{D}_{p}\right)$ for all primes $p$ but is not residually finite since it contains a subgroup isomorphic to the additive group of all rational numbers.

Now we show that nondivisibility conditions are not stable under infinite extensions by cyclic groups.

EXAMPLE 4.4. Let $H=G(\phi) \rtimes\langle g\rangle$ be the semidirect product of the group $G(\phi)$ from Example 4.3 and the infinite cyclic group generated by $g$ such that $g z=z g, g y=y g$ and $g^{-1} x g=x^{2}$. Then $x$ is conjugate in $H$ to $x^{p}$ for all primes $p$. Hence the group $H$ does not satisfy any of the conditions (WD), (D), (*) or $\left(\mathrm{D}_{p}\right)$. 
Proposition 4.5. The following classes of groups are closed under subdirect products:

(i) $\left(\mathrm{D}_{p}\right)$-groups having at most p-torsion.

(ii) Torsion free (D)-groups.

(iii) (WD)-groups.

Proof. Since proofs of all parts are similar we only show (i). Let $G \subseteq$ $\prod_{j \in J} G_{j}$ be a subdirect product of $\left(\mathrm{D}_{p}\right)$-groups with $p$-torsion only. Let $g=$ $\left(g_{j}\right), h=\left(h_{j}\right) \in G$ be such that $h^{-1} g h=g^{p^{N}}$. Then for each $j, h_{j}^{-1} g_{j} h_{j}=$ $g_{j}^{p^{N}}$ so $g_{j}=1$. Hence $g=1$.

In [1] Bass wrote: "We do not know whether all residually finite groups satisfy condition (D)". The negative answer was given by Wilson [11]. Now we present a new construction of such "bad" groups.

THEOREM 4.6. Let $m>1$. Let $\phi: \mathbb{Z} \rightarrow \mathbb{Z}$ be defined by $\phi(i)=i m+1$. Then $G(\phi)$ satisfies the condition (WD) but does not satisfy (D) or (*). Moreover, for each prime $p$ the following conditions are equivalent:

(i) $p$ divides $m$.

(ii) $G(\phi)$ is approximated by finite p-groups.

(iii) $G(\phi)$ satisfies condition $\left(\mathrm{D}_{p}\right)$.

Proof. The implication (i) $\Rightarrow$ (ii) follows from Theorem 2.7.

(ii) $\Rightarrow$ (iii) follows from Proposition 4.5.

(iii) $\Rightarrow($ i). Take a prime $q$ such that $q$ does not divide $m$. Since at least two of the integers $1, q, q^{2}, \ldots, q^{m}$ are congruent modulo $m, m$ divides $q^{m !}-1$ so $q^{m !}$ has the form $i m+1$. Hence $G(\phi)$ does not satisfy $\left(\mathrm{D}_{q}\right)$.

Furthermore, by Theorem 4.1(ii), $q^{m !}-1 \in C$ for primes $q>m$ implies that $G(\phi)$ satisfies neither (D) nor $(*)$.

\section{REFERENCES}

[1] H. Bass, Euler characteristics and characters of discrete groups, Invent. Math. 35 (1976), 155-196.

[2] S. Dăscălescu, A note on groups with the finite embedding property, Proc. Internat. Conf. on Group Theory (Timişoara, 1992), An. Univ. Timişoara Ser. Ştiinţ. Mat. 1993, special issue, 43-45.

[3] S. Dăscălescu, C. Năstăsescu, A. del Rio and F. Van Oystayen, Gradings of finite support. Application to injective objects, J. Pure Appl. Algebra 107 (1996), 193-206.

[4] E. Formanek, Idempotents in Noetherian group rings, Canad. J. Math. 15 (1973), 366-369.

[5] P. Hall, On the finiteness of certain soluble groups, Proc. London Math. Soc. (3) 9 (1959), 595-622.

[6] I. Kaplansky, Problems in the theory of rings, in: Report of a Conference on Linear Algebras, National Acad. Sci., Washington, 1957, 1-3. 
[7] P. A. Linnel, Decomposition of augmentation ideals and relation modules, Proc. London Math. Soc. 47 (1983), 83-127.

[8] A. V. Mikhalev and A. E. Zalesskiŭ, Group Rings, Nauka, Moscow, 1973 (in Russian).

[9] D. J. S. Robinson, Finiteness Conditions and Generalized Soluble Groups, Springer, Berlin, 1972.

[10] A. Strojnowski, On Bass' "Strong Conjecture" about projective modules, J. Pure Appl. Algebra 62 (1989), 195-198.

[11] J. S. Wilson, Embedding theorems for residually finite groups, Math. Z. 174 (1980), 149-157.

Institute of Mathematics

Warsaw University

Banacha 2

02-097 Warszawa, Poland

E-mail: stroa@mimuw.edu.pl

Received 17 January 1998;

revised 6 May 1998 